\title{
Réformes et enjeux de l'enseignement supérieur en Bulgarie
}

\section{Gilles Rouet}

\section{OpenEdition}

1 Journals

Édition électronique

URL : https://journals.openedition.org/ries/2657

DOI : 10.4000/ries.2657

ISSN : 2261-4265

Éditeur

France Education international

Édition imprimée

Date de publication : 1 décembre 2012

Pagination : 25-32

ISSN : 1254-4590

\section{Référence électronique}

Gilles Rouet, «Réformes et enjeux de l'enseignement supérieur en Bulgarie », Revue internationale d'éducation de Sèvres [En ligne], 61 | décembre 2012, mis en ligne le 06 février 2015, consulté le 05 juillet 2021. URL : http://journals.openedition.org/ries/2657 ; DOI : https://doi.org/10.4000/ries.2657

Ce document a été généré automatiquement le 5 juillet 2021.

(c) Tous droits réservés 


\title{
Réformes et enjeux de l'enseignement supérieur en Bulgarie
}

\author{
Gilles Rouet
}

1 Depuis 1989, la Bulgarie poursuit une politique de rapprochement avec l'Ouest et a multiplié les alliances politiques (OTAN en 2002, Organisation internationale de la francophonie en 2003, Union européenne en 2007), les gouvernements successifs considérant que l'intégration européenne est la seule option envisageable pour le développement et la modernisation du pays. En particulier, l'ambition est d'améliorer le secteur de l'éducation. Il s'agit de « rattraper l'Ouest».

2 Le contexte est cependant difficile. Dans ce pays de 7,4 millions d'habitants, les effets conjugués, principalement, de la dénatalité et des migrations économiques ont abouti à une chute de la démographie ( $-1,4$ million d'habitants depuis 1989), en partie compensée, pour l'enseignement supérieur, par l'augmentation de la demande de formation ${ }^{1}$. Le taux net de scolarisation dans l'enseignement supérieur est désormais supérieur à $41 \%$ de la classe d'âge 19-23 ans, ce qui implique une augmentation des taux d'encadrement, une baisse des coûts par étudiant de l'enseignement supérieur mais aussi une plus grande hétérogénéité des étudiants à prendre en compte.

La rigueur budgétaire imposée par le processus d'intégration européenne, l'évasion et la fraude fiscales ainsi que la corruption amènent les gouvernements à opérer des choix, à contraindre les budgets publics et à chercher des sources de financement alternatives (y compris auprès des étudiants, malgré le faible niveau général des pouvoirs d'achat). Les acteurs de l'enseignement supérieur interprètent souvent les réformes comme des tentatives de démembrement du secteur public et de marchandisation de l'éducation et sont de plus très peu impliqués dans la mise en place des réformes. Une crispation des rapports entre le gouvernement et les responsables des universités a abouti de facto à un statu quo dangereux qui contrarie toute évolution positive. 
4 Les établissements ont peu de moyens et les professions universitaires restent peu attractives en termes de revenu ${ }^{2}$ (comme, d'ailleurs, en termes de reconnaissance sociale, les intellectuels n'ayant pas le sentiment de "compter » dans le pays), ce qui favorise la poursuite de la fuite des cerveaux désormais moins visible, d'une part car elle est orientée vers l'Europe de l'Ouest plutôt que vers les États-Unis ${ }^{3}$, d'autre part parce que les jeunes adoptent de plus en plus un comportement « mobile» et non plus " migrant ", effectuant de multiples voyages entre leur domicile bulgare et leur lieu de travail européen.

\section{Politique et organisation de l'enseignement supérieur}

5 Après la création de la première université bulgare lors de la "Renaissance bulgare "4, le système d'enseignement supérieur a évolué principalement sous influence européenne (dont russe) puis soviétique après 1945. La dernière grande loi sur l'enseignement supérieur a été promulguée le 12 décembre 1995 et accorde en particulier une très grande autonomie académique aux établissements. Elle permet le passage d'un diplôme à un autre (pour rompre avec le caractère «tubulaire » des études du système précédent) et la création de différents niveaux de sorties. Cette loi a été complétée en 1999 puis modifiée régulièrement, notamment pour accroître l'autonomie des établissements en matière de gestion financière ce qui, dans un contexte de crise économique et de réduction budgétaire, ne convient pas à tous les acteurs du système.

6 En 2009 a été mise en place une logique d'accréditation des établissements en trois catégories : universités, collèges universitaires et écoles supérieures et, en 2010, la Commission supérieure d'accréditation (VAK) a été supprimée. Les titres universitaires sont désormais délivrés par les établissements (doctorat, mais aussi qualifications aux fonctions de maître de conférences habilité - docent - ou de professeur), ce qui a donné lieu à un débat important sur la qualité et le contrôle des nominations, désormais liées aux stratégies de chaque établissement. Beaucoup craignent la multiplication de professeurs non crédibles et se souviennent de certaines nominations de l'époque soviétique.

7 Le système universitaire bulgare est a priori sélectif à l'entrée, même si le nombre de candidats dans certaines filières et le développement de l'enseignement privé relativisent cette sélectivité. L'accès aux universités se fait par concours d'entrée (différents d'une université à l'autre) ou bien avec la prise en compte des résultats obtenus à la matura (diplôme de fin d'études secondaires obligatoire depuis juin 2008), selon l'image de qualité dont bénéficie chaque université auprès du public, la démographie et la politique de l'établissement. Ainsi, à côté d'un effet "réputation » des universités et filières, existe désormais une démarche de réponse à une demande de formation, en particulier dans les domaines de l'économie, du management et du droit, alors que le nombre d'universitaires susceptibles d'enseigner dans ces champs reste contraint (suite à la « fuite des cerveaux »).

8 Le système s'articule en trois cycles: un premier cycle de trois ou quatre années d'études (bakalavar professionnel ou bakalavar), un deuxième cycle de deux à quatre semestres intégrant très souvent suivi un stage de six mois (master - magistăr -, la plupart en trois semestres) et les études doctorales, trois à cinq années après le magistăr. Les études de médecine, médecine vétérinaire, architecture ou droit, mènent 
directement à un master après cinq ans d'études. La structure des études fait débat dans le pays car la durée de la plupart des masters ne facilite pas les coopérations européennes. Est également discutée la pertinence de la durée de quatre ans du premier cycle mais il semble difficile d'envisager d'adopter une structure de type $3+2$, non seulement à cause des mécanismes d'insertion professionnelle (et même si le nombre d'étudiants souhaitant poursuivre en master augmente régulièrement) mais aussi parce que l'adoption d'une architecture 3+2 aboutirait, de facto, à l'augmentation d'une année d'études pour la grande majorité des étudiants, ce qui n'est pas tenable budgétairement. Enfin, les universitaires sont réticents à généraliser les masters en deux semestres (et donc à $4+1$ ), ce qui ne donne pas la possibilité d'un stage long (même si, étant donné qu'entre 40 et $60 \%$ des étudiants à ce niveau ont un travail salarié, des aménagements devraient être possibles). L'intégration de ce stage dans les études est un élément fondamental de modernisation, alors même que peu d'établissements organisent un suivi et une évaluation pédagogiques. En fait, le maintien de filières tubulaires en cinq ans facilite a contrario les coopérations universitaires et favorise les doubles diplômes, ce qui explique que plusieurs facultés des universités techniques aient adopté cette architecture.

La Bulgarie dispose d'un grand nombre d'établissements d'enseignement supérieur au regard de sa population et du nombre total de ses étudiants. Ainsi, plus des deux tiers des établissements ont moins de mille étudiants. D'après les estimations, entre 25000 et 80000 étudiants bulgares sont inscrits à l'étranger, selon que l'on prend en compte les étudiants bulgares en poursuite d'études à l'étranger ou bien les enfants des familles installées à l'étranger devenus étudiants dans le pays d'accueil ${ }^{5}$.

L'enseignement supérieur en Bulgarie est assuré par les universités et écoles supérieures publiques, les académies d'État et par des établissements privés qui doivent désormais être accrédités par la commission nationale qui reconnaît trois statuts: universités, écoles d'enseignement supérieur et collèges. Les universités assurent des formations dans tous les domaines et gèrent aussi des équipes de recherche. La plupart des instituts ou académies, au départ établissements supérieurs autonomes spécialisés dans un secteur particulier, sont progressivement devenus des universités. Les collèges, autonomes ou intégrés à une université, proposent des formations plus courtes, à vocation professionnelle (les bakalavars professionnels).

11 La Bulgarie, qui a signé la déclaration de Bologne dès 1999, est intégrée au comité de suivi du processus et a procédé à une réorganisation institutionnelle pour renforcer les mécanismes d'évaluation (création de l'Agence nationale d'évaluation et d'accréditation : NAOA) ${ }^{6}$, se rapprocher de l'Association européenne pour l'assurance qualité (ENQA) et s'inscrire au Registre de l'assurance qualité (EQAR). Cependant, la structure des cursus de 1995 (4-5/6-8/11) n'a pas évolué.

Le système de crédits européens ECTS a été généralisé en 2004-2005 mais il rencontre des difficultés à être opérationnel car beaucoup d'étudiants ont encore du mal à faire reconnaître les crédits acquis à l'étranger dans le cadre des échanges européens, y compris dans le cadre du programme Erasmus ${ }^{7}$. Le Processus de Bologne semble considéré par la plupart des acteurs comme une réforme institutionnelle lié à l'adhésion à l'Union européenne ${ }^{8}$, un outil de "rattrapage ", et les instruments ont souvent été implantés par transformation du système existant, comme les ECTS mais aussi le Supplément au diplôme, dont le contenu, très généralement, ne s'articule pas avec une description en acquisition de compétences des formations. 


\section{Le difficile financement de l'enseignement supérieur}

13 ministère de l'Éducation, de la Jeunesse et de la Science et le financement public est calculé en fonction du nombre d'étudiants depuis 1999. Depuis 2010, le gouvernement cherche à modifier le système de calcul et tente de réduire la part de financement public des établissements d'enseignement supérieur publics (65\% du financement total), en augmentant les droits de scolarité et en incitant à la recherche d'autres sources de financement. La loi autorise les établissements à fixer librement les droits d'inscription, dans la limite de $30 \%$ de la dotation versée par l'État pour les places subventionnées (dont le nombre varie en fonction des spécialités), voire de façon totalement libre pour les établissements les mieux notés par l'Agence nationale d'évaluation et d'accréditation. Le gouvernement définit le nombre d'étudiants susceptibles d'être admis pour chaque programme et pris en charge par le budget de l'État mais, suite aux mesures d'austérité consécutives à la crise, le gouvernement permet désormais aux établissements publics d'inscrire des étudiants à tous niveaux en contrepartie du paiement intégral des coûts de scolarité et à condition que le nombre d'étudiants inscrits ainsi ne dépasse pas $10 \%$ de la capacité de l'institution. Comme l'augmentation des frais de scolarité est en contradiction avec la garantie par l'État de l'accès aux études supérieures, des bourses sous condition de ressources complètent celles sur le mérite et le gouvernement a lancé un programme de prêts pour les étudiants 9 .

Certaines universités (techniques en particulier) développent des coopérations avec des entreprises et réalisent des travaux d'expertise dont la rétribution est intégrée au moins en partie dans le budget des établissements. Néanmoins, les procédures financières restent complexes et peu flexibles et de nombreux départements ou facultés ont créé des personnalités morales de droit privé (association, ONG) pour recevoir des fonds extérieurs ou gérer des projets particuliers. Cette situation n'est évidemment pas susceptible d'améliorer les situations budgétaires des établissements.

Les universités privées en Bulgarie ne reçoivent aucune dotation financière de l'État et doivent donc assumer elles-mêmes la totalité des coûts. Les établissements privés sont, comme le secteur public, soumis aux exigences légales. Ils accueillent plus de $18 \% \mathrm{du}$ total des étudiants et se répartissent en trois catégories : privés " profitables ", en fait des business school souvent de qualité qui attirent également des étudiants étrangers; privés «à but non lucratif $»^{10}$, animés par des universitaires souvent issus du secteur public (la rémunération pouvant être doublée voire triplée) et financés en partie par des fondations ${ }^{11}$; des établissements non accrédités en Bulgarie (et donc en relative illégalité) ${ }^{12}$. Les droits de scolarité dans la première catégorie sont élevés (3 à 10000 euros par an selon les établissements) tandis que les droits dans la deuxième catégorie restent relativement faibles (500 euros environ par semestre à la Nouvelle université bulgare) en comparaison des droits de scolarité dans le secteur public, qui augmentent depuis plusieurs années (entre 100 et 300 euros par semestre en moyenne ${ }^{13}$ ). Si cette tendance se poursuit, le différentiel de montant des droits de scolarité entre le public et une partie du privé deviendra de moins en moins important, alors qu'objectivement les conditions d'études sont bien plus confortables au sein des (bonnes) universités privées.

Revue internationale d'éducation de Sèvres, 61 | décembre 2012 


\section{Bilan et défis} augmente l'inertie et les conservatismes, et compte tenu d'un manque de contrôle central qui encourage les adaptations locales, les clientélismes et l'abandon de programmes de formation "peu rentables» en termes de nombre d'étudiants. La grande tradition de l'enseignement technologique, l'un des points forts du système bulgare continuent heureusement d'attirer de nombreux étudiants, les débouchés étant toujours réels.

19 Il y aurait trop d'étudiants en Bulgarie: plus de $60 \%$ des diplômés du secondaire s'inscrivent en effet dans l'enseignement supérieur. Si cette affirmation est conforme à l'analyse économique de la situation, elle ne prend pas en compte les logiques sociales et surtout, elle va à contre-courant de la volonté affichée d'intégration européenne et de modernisation de la société. La Bulgarie étant le seul pays des vagues d'adhésion 2004 et 2007 à n'avoir pas prévu de programme opérationnel pour utiliser les fonds structurels pour l'enseignement et la recherche, le gouvernement cherche actuellement à mobiliser cette manne financière européenne notamment pour l'enseignement supérieur, sans pour autant remettre en cause les autres programmes prévus pour 2014-2020 et, surtout, sans concertation organisée.

20 À moyen terme, pour 2015, le gouvernement prévoit de poursuivre la «mise à jour » réglementaire (plus d'autonomie des établissements), d'établir un ranking des universités bulgares mobilisable pour le calcul des subventions d'État, de mettre en place des procédures d'organisation et de suivi des stages des étudiants, de faire évoluer le système des bourses (dans un souci d'égalité d'accès) et de créer une structure de formation et de perfectionnement professionnel des enseignants.

21 Ces évolutions ne régleront pour autant pas le problème de sous-financement de l'enseignement supérieur, aggravé par la faible performance des établissements en matière de collecte de fonds. Il apparaît que l'augmentation du financement public de l'enseignement supérieur, à hauteur de $1 \%$ du PIB, est désormais nécessaire mais 
difficile $^{14}$ et devrait s'accompagner d'une réforme des modalités de subventionnement de financement des établissements, en particulier, à l'instar de l'évolution française dans ce domaine, avec une modification des critères (par exemple nombre de diplômés au lieu de nombre d'inscrits). L'introduction d'une logique de performance est également envisagée. Les pouvoirs politiques comme les acteurs de l'enseignement supérieur reconnaissent généralement que le nombre d'établissements est trop important. Des projets de fusion ont été initiés, sans concrétisation pour le moment (notamment la création d'une grande université technologique à Sofia), à la fois pour améliorer la performance en matière de recherche et pour faciliter l'apport de ressources extérieures ${ }^{15}$.

En définitive, le pays doit trouver dans ses propres ressources, surtout humaines, la façon d'établir une synergie entre enseignement et recherche au sein des établissements et dépasser les dogmes (néolibalisme vs démocratisme social) pour trouver les moyens d'une coopération entre les secteurs privé et public. Encore faudrat-il, pour cela, que le processus de démocratisation de la société se poursuive et que chaque citoyen, chaque acteur, se sente concerné par les institutions et leur avenir et agisse en conséquence.

\section{BIBLIOGRAPHIE}

DOBBINS M (2011) : Higher Education Policies in Central and Eastern Europe: Convergence towards a Common Model? Palgrave Macmillan.

GEORGIEVA P., PILEV D. and TODOROVA L. (2002) : Higher Education in Bulgaria. UNESCO-CEPES Monographs on Higher Education. Bucarest.

MITRA P. \& POUVELLE C. (2012) : Productivity Growth and Structural Reform in Bulgaria: Restarting the Convergence Engine, Fonds Monétaire International.

Republic of Bulgaria (2011) : Ministry of Education, Youth and Science, National Report, Contribution to the Joint Progress Report of the Council and the Commission on the Implementation of the Strategic Frame-work for European Cooperation in Education and Training Education 2020, http://ec.europa.eu/education/lifelong-learning-policy/doc/ natreport11/bulgaria_en.pdf/.

Republic of Bulgaria (2011) : National Reform Programme (2011-2015), in implementation of "Europe 2020" Strategy, http://ec.europa.eu/europe2020/pdf/nrp/nrp_bulgaria_en.pdf/.

TOTOMANOVA A (2002) : The role of Bulgarian universities in the transformation of society: Case Study. Centre for Educational Strategies, Sofia. 


\section{ANNEXES}

\section{Quelques statistiques}

- 53 établissements d'enseignemant supérieur, dont 9 collèges indépendants

( 22 collèges sont intégrés aux universités) ;

- 487 établissements d'enseignement secondaire ;

$-55,9 \%$ de la population bulgare parle au moins une langue étrangère ;

$-70 \%$ des étudiants étudient l'anglais.

Source: http://investbg.government.bg/en

Étudiants et enseignants

\begin{tabular}{|l|c|c|}
\cline { 2 - 3 } \multicolumn{1}{c|}{} & Année 2011/2012 & Évolution depuis 2006 (\%) \\
\hline Nombre total d'étudiants & $\mathbf{2 8 0 2 9 2}$ & $+\mathbf{1 0}$ \\
\hline Licence professionnelle & $16210^{1}$ & -41 \\
\hline Licence et master & 264082 & +16 \\
\hline Doctorat & 4703 & +2 \\
\hline Étudiants du secteur privés & 52586 & +4 \\
\hline Étudiants étrangers & 10281 & $+1,6$ \\
\hline Dont licence et master & 10024 & +8 \\
\hline Personnel académique & $\mathbf{2 2 9 5 5}$ & $-3,5$ \\
\hline Dont professeurs & 2576 & $+0,8$ \\
\hline Dont docent (maîtres de conférences habilités) & 7139 & $-12,5$ \\
\hline Dont maîtres-assistants & 9881 & 3350 \\
\hline Dont assistants & . & \\
\hline
\end{tabular}

1. Plusieurs collèges sont devenus des universités en 2010 et leurs «bakalavars professionnels » ont ainsi été reconnues comme «bakalavars», ce qui explique l'importante baisse des effectifs. 
Répartitions des étudiants selon les champs d'études

\begin{tabular}{|l|r|r|r|r|}
\hline Champs d'études & $\mathbf{2 0 0 6 / 2 0 0 7}$ & $\mathbf{2 0 1 1 / 2 0 1 2}$ & $\begin{array}{c}\text { Répartition } \\
\mathbf{2 0 1 1}(\mathbf{\%})\end{array}$ & $\begin{array}{c}\text { Évolution } \\
\text { (\%) }\end{array}$ \\
\hline Total & 253876 & 280292 & & 10,41 \\
\hline Management & 64362 & 66304 & 23,66 & 3,02 \\
\hline Sciences de l'ingénieur & 39474 & 39810 & 14,20 & 0,85 \\
\hline Sciences sociales & 34233 & 33049 & 11,79 & $-3,46$ \\
\hline Education & 16096 & 18337 & 6,54 & 13,92 \\
\hline Santé & 12786 & 18872 & 6,73 & 47,60 \\
\hline Sciences humaines & 14112 & 13983 & 4,99 & $-0,91$ \\
\hline Droit & 10474 & 11263 & 4,02 & 7,53 \\
\hline Architecture et construction & 6446 & 9717 & 3,47 & 50,74 \\
\hline Informatique & 6697 & 8651 & 3,09 & 29,18 \\
\hline Services aux personnes & 10823 & 10765 & 3,84 & $-0,54$ \\
\hline Arts & 5627 & 7086 & 2,53 & 25,93 \\
\hline Services de sécurité & 4783 & 6643 & 2,37 & 38,89 \\
\hline Agriculture et forets & 4876 & 5060 & 1,81 & 3,77 \\
\hline Techniques & 3963 & 4313 & 1,54 & 8,83 \\
\hline Physiques et chimie & 3512 & 3779 & 1,35 & 7,60 \\
\hline Journalisme et communication & 3842 & 3670 & 1,31 & $-4,48$ \\
\hline Protection de l'environnement & 2715 & 3601 & 1,28 & 32,63 \\
\hline Transports & 2158 & 2922 & 1,04 & 35,40 \\
\hline Services sociaux & 2822 & 2336 & 0,83 & $-17,22$ \\
\hline Sciences vétérinaires & 1446 & 1453 & 0,52 & 0,48 \\
\hline Sciences de la vie & 1289 & 1406 & 0,50 & 9,08 \\
\hline Mathématiques et statistiques & 888 & 590 & 0,21 & $-33,56$ \\
\hline & & & & \\
\hline
\end{tabular}

\section{NOTES}

1. 19,6\% de la population a suivi des études supérieures, 43,4\% des études secondaires (2010).

2. Pour un assistant, en moyenne 150 euros par mois, pour un professeur en fin de carrière, entre 450 et 500 euros au sein des universités publiques. Un docent perçoit entre 300 et 400 euros mensuels nets. La faiblesse des rémunérations incite beaucoup d'enseignants à multiplier les activités, au sein ou en dehors du système universitaire, pour compléter leurs revenus (et notamment dans le cadre des fondations ou de programmes européens).

3. Un inventaire réalisé en 2012 montre qu'au moins les deux tiers des boursiers du gouvernement français, depuis vingt-cinq ans, ne sont pas revenus en Bulgarie.

4. L'Université de Sofia Saint Clément d'Ohrid a été créée en 1888.

5. Environ 8000 étudiants bulgares sont en Allemagne, plus de 4000 en Autriche, environ 2500 en France, 3000 aux États-Unis, 1500 en Grèce et moins de 500 en Grande-Bretagne.

6. Tous les établissements doivent faire habiliter leurs diplômes et formations par cette Agence qui a, depuis sa création, évalué tous les établissements publics d'enseignement supérieur de Bulgarie et la plupart des privés. Si un établissement public est évalué négativement, un délai lui est donné pour tenter de remédier à la situation.

7. Ce qui est cependant facilité par le mode d'évaluation des étudiants et les procédures d'examen déterminées par chaque établissement. En général est utilisée, à partir d'examens écrits, une échelle allant de 1 à 6 mais certaines universités utilisent aussi des scores intermédiaires de 5,5 ou de 4,5 .

8. Voir Radovan Gura et Gilles Rouet, Les Universités en Europe centrale, 20 ans après, vol. 1, Evolutions et enjeux, Ludmila. Meskova et Thierry Come, vol. 2, Économie et management, Bruxelles, Bruylant, 2011 ainsi que Thierry Côme et Gilles Rouet, Processus de Bologne, construction européenne, politique européenne de voisinage, Bruxelles, Bruylant, 2011. 
9. En 2011, 4800 étudiants (moins de $2 \%$ ) ont obtenu un prêt et environ $6 \%$ (16 000 étudiants) bénéficiaient d'une bourse d'État.

10. Comme la Nouvelle université bulgare à Sofia, qui compte plus de 13000 étudiants et une soixantaine de spécialités.

11. Dont des fondations créées par des Bulgares émigrés ayant fait fortune à l'étranger pendant l'époque soviétique.

12. Une vingtaine, d'après le président de l'agence d'accréditation.

13. En sciences de l'ingénieur, le montant des droits peut représenter plus du double. En médecine ou pour les études de beaux-arts, les coûts d'inscription sont environ cinq fois plus élevés. Les droits d'inscription pour les étudiants étrangers (hors Union européenne) sont au minimum, d'après la loi, de 3000 euros par an et les formalités d'inscriptions (comme pour les étudiants de l'Union européenne) restent assez complexes.

14. Taux en 2010, 0,8 \% (source : http://www.eurocompar.eu/).

15. Il s'agit d'une des préconisations de la Banque mondiale, comme l'autonomie des établissements.

INDEX

Mots-clés : enseignement supérieur, financement, réforme de l'enseignement, université

Palabras claves : enseñanza superior, financiación, reforma de la educación, universidad Index géographique : Bulgarie

Keywords : higher education, financing, educational reform, universities

\section{AUTEUR}

\section{GILLES ROUET}

Professeur de sciences de l'éducation à l'Université de Reims, membre du GEPECS, Université Paris Descartes, garant scientifique des études d'études européennes culturelles à l'Université Matej Bel de Banska Bystrica (Slovaquie) depuis 2009, Chaire Jean Monnet ad personam en études interdisciplinaires sur l'Union européenne et attaché de coopération universitaire et scientifique en Slovaquie (2004-2008) et en Bulgarie (depuis décembre 2010) 